\title{
Detailed FSI modeling and HPC simulation of the Olympic stadium roof in Munich under wind loading
}

\author{
Philipp Bucher*, Máté Péntek, Klaus Bernd Sautter, Roland Wüchner, Kai-Uwe \\ Bletzinger
}

Chair of Structural Analysis

Technische Universität München (TUM)

Arcisstr. 21, D-80333 München, Germany

\begin{abstract}
The Olympic stadium in Munich was built for the Olympic Games in 1972. Its architecture was inspired by freefrom shapes and led to the construction of "hanging" cable nets covered with acrylic glass plates, which constitute the main part of the roof. The main structure additionally includes pylons, masts, and cables to hold up this complex geometry. Details of the construction are described in [1].

The main focus of this work is to improve the numerical models and showcase the simulation potential for such a complex geometry, with a specific focus on the realistic modeling of the wind field (atmospheric boundary layer flow and consideration of some crucial elements of topography) as well as fluid-structure interaction (FSI, i.e. enabling the interaction between the deformations of the structure and the wind forces adapting to these changes). For the fluid domain, only the roof is explicitly captured by the mesh, in addition to the chosen topography elements. Other parts of the supporting structure are deemed to have a minimal role in the aerodynamics.
\end{abstract}

Careful considerations are taken regarding the modeled geometry of the structure, these being based upon [2], data extracted from a Google Earth model, on-site measurements, and city archives. This shape represents the target geometry under self-weight and prestress. The membrane, as well as cable elements (modeled as trusses, being purely under tension), need a detailed investigation such that the correct prestress values can be determined. Initial values are based upon archive and publication data ([1]). Optimization algorithms are used in a sequential way to determine the actual necessary prestress (on an element level in the FEM context). This is to be seen as an inverse of the fromfinding procedure.

An advanced coupling framework is used to ensure the proper communication between the fluid and structural domains. This is done in a two-way fashion, meaning that structural displacements and fluid loads are exchanged at each time step. The applied partitioned approach implies that the fluid and structural models and solvers need to be set up both individually in a precise manner as well as additional steps are taken to make sure the physical interaction can be handled properly by the numerical scheme. Dedicated mapping further enables the transfer of the relevant quantities (aerodynamic forces and structural deformation) between the non-matching numerical meshes.

Challenges, as well as opportunities, are provided by the supercomputing (HPC) environment used to perform this advanced analysis. The open-source multi-physics FEM framework Kratos Multiphysics [3] hosts the developments necessary to enable such simulations. The various components (fluid, structure, coupling, mapping) are implemented in a modular way, besides complying with the requirements for scalability on a large distributed memory infrastructure.

* Corresponding author. E-mail: philipp.bucher@tum.de

\section{REFERENCES}

[1] Leonhardt F., Schlaich J., Weitgespannte Flächentragwerke, Sonderforschungsbereich 64, University of Stuttgart (1973) 
[2] Cotela-Dalmau J., Bucher P., Ghantasala A., Andre M., Winterstein (geb. Mini) A., Rossi R. and Wüchner R., Implementation of mapping strategies in a distributed memory environment, VII International Conference on Coupled Problems in Science and Engineering (2017).

[3] Dadvand, P., Rossi, R. and Oñate, E., An Object-oriented Environment for Developing Finite Element Codes for Multi-disciplinary Applications, Archives of Computational Methods in Engineering 17 (2010) 253-297.
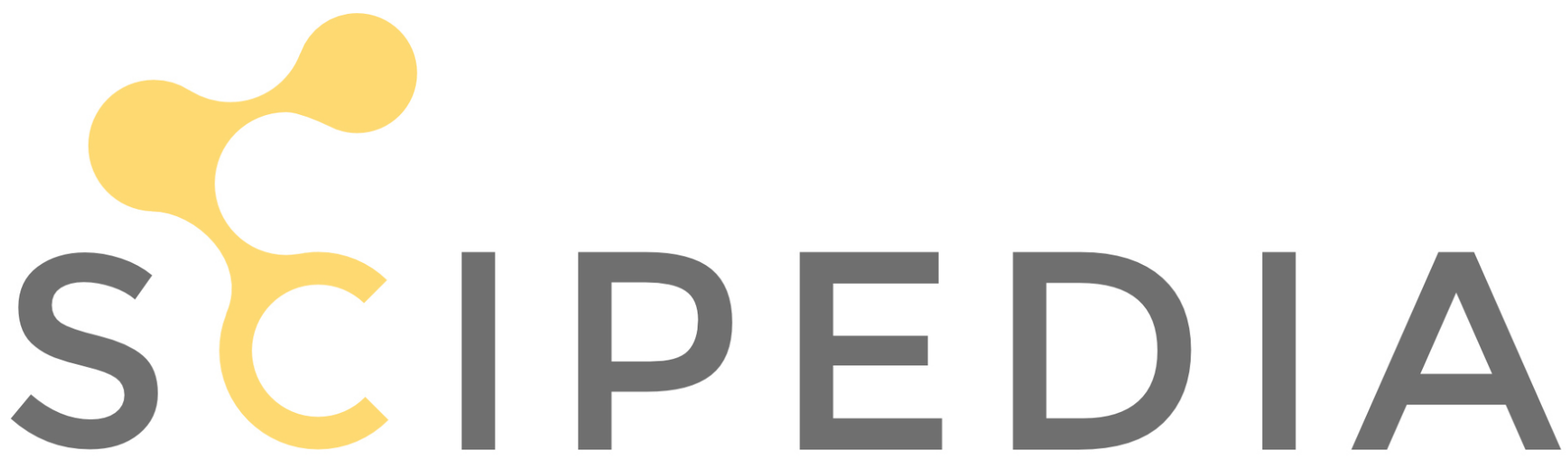

Register for free at https//www.scipedia.com to download the version without the watermark 